\title{
Modeling and gene knockdown to assess the contribution of nonsense-mediated decay, premature termination, and selenocysteine insertion to the selenoprotein hierarchy
}

\author{
ANZE ZUPANIC, ${ }^{1,2,5}$ CATHERINE MEPLAN, ${ }^{1,3,5}$ GRAZIELLE V.B. HUGUENIN, ${ }^{3,4}$ JOHN E. HESKETH, ${ }^{1,3,6}$ \\ and DARYL P. SHANLEY ${ }^{1,3,6}$ \\ ${ }^{1}$ Centre for Integrated Systems Biology of Ageing and Nutrition, Newcastle University, Newcastle-upon-Tyne NE4 5PL, \\ United Kingdom \\ ${ }^{2}$ Eawag, Institute for Aquatic Science and Technology, 8600 Dübendorf, Switzerland \\ ${ }^{3}$ Institute for Cell and Molecular Biosciences and Human Nutrition Research Centre, Newcastle University, \\ Newcastle-upon-Tyne NE2 4HH, United Kingdom \\ ${ }^{4}$ Faculty of Medicine, Federal University of Rio de Janeiro, Rio de Janeiro, CEP: 21941-902, Brazil
}

\begin{abstract}
The expression of selenoproteins, a specific group of proteins that incorporates selenocysteine, is hierarchically regulated by the availability of Se, with some, but not all selenoprotein mRNA transcripts decreasing in abundance with decreasing Se. Selenocysteine insertion into the peptide chain occurs during translation following recoding of an internal UGA stop codon. There is increasing evidence that this UGA recoding competes with premature translation termination, which is followed by nonsense-mediated decay (NMD) of the transcript. In this study, we tested the hypothesis that the susceptibility of different selenoprotein mRNAs to premature termination during translation and differential sensitivity of selenoprotein transcripts to NMD are major factors in the selenoprotein hierarchy. Selenoprotein transcript abundance was measured in Caco-2 cells using real-time PCR under different Se conditions and the data obtained fitted to mathematical models of selenoprotein translation. A calibrated model that included a combination of differential sensitivity of selenoprotein transcripts to NMD and different frequency of non-NMD related premature translation termination was able to fit all the measurements. The model predictions were tested using SiRNA to knock down expression of the crucial NMD factor UPF1 (up-frameshift protein 1) and selenoprotein mRNA expression. The calibrated model was able to predict the effect of UPF1 knockdown on gene expression for all tested selenoproteins, except SPS2 (selenophosphate synthetase), which itself is essential for selenoprotein synthesis. These results indicate an important role for NMD in the hierarchical regulation of selenoprotein mRNAs, with the exception of SPS2 whose expression is likely regulated by a different mechanism.
\end{abstract}

Keywords: selenoprotein; mathematical modeling; translation; nonsense-mediated decay; premature termination

\section{INTRODUCTION}

The micronutrient selenium (Se) is essential for health (Rayman 2012) and its biological functions are brought about through selenoproteins, a specific group of proteins that incorporate selenocysteine, the 21st amino acid (Labunskyy et al. 2014). Selenocysteine insertion into the peptide chain occurs during translation and follows a recoding of an internal premature termination (UGA) codon (PTC) as selenocysteine, which requires a specific stemloop structure (called the SECIS) within the $3^{\prime}$ untranslated

\footnotetext{
${ }^{5}$ These authors contributed equally to this work.

${ }^{6}$ These authors contributed equally to this work.

Corresponding author: daryl.shanley@ncl.ac.uk

Article published online ahead of print. Article and publication date are at http://www.rnajournal.org/cgi/doi/10.1261/rna.055749.115. Freely available online through the RNA Open Access option.
}

region ( $3^{\prime} \mathrm{UTR}$ ) of selenoprotein mRNAs (Copeland et al. 2000; Kryukov et al. 2003; Latrèche et al. 2009). It is widely assumed that recoding of the UGA codon competes with premature translation termination, which is followed by nonsense-mediated decay (NMD), a process that targets aberrant transcripts with PTCs for degradation (Berry et al. 1994; Sun et al. 2001). The expression of selenoproteins is also selectively regulated by the availability of Se, and therefore of selenocysteine, with some, but not all selenoprotein mRNAs increasing in abundance with increased availability of $\mathrm{Se}-\mathrm{a}$ phenomenon that has been named the selenoprotein hierarchy (Wingler et al. 1999; Low et al. 2000; Fletcher et al. 2001). The precise mechanism(s) underlying

(C) 2016 Zupanic et al. This article, published in $R N A$, is available under a Creative Commons License (Attribution 4.0 International), as described at http://creativecommons.org/licenses/by/4.0/. 
the hierarchy is not fully understood, although it is likely that differences in the $3^{\prime}$ UTR sequence of the different mRNAs are involved in determining competition between the mRNAs for available selenocysteine tRNA and therefore the extent to which the different mRNAs are translated.

One possibility is that different susceptibility of selenoprotein mRNAs to NMD is involved in determining the hierarchical regulation of the selenoprotein mRNAs with decreasing selenocysteine tRNA. A recent study has provided some experimental support for this hypothesis (Seyedali and Berry 2014) and earlier work showed that during Se deficiency, GPX1 mRNA, but not GPX4 mRNA, was targeted by NMD (Weiss and Sunde 1997; Moriarty et al. 1998). However, there is also evidence that susceptibility of NMD is not sufficient to explain the variability of selenoprotein mRNA responses to Se deficiency. A currently accepted mechanism/hypothesis proposes that only transcripts featuring a PTC of at least 50-55 nucleotides (nt) upstream of an exon junction are vulnerable to NMD (Popp and Maquat 2013). On this basis, SELK should not be a target of NMD, while TXNRD2 should be. However, a recent study of dietary Se effects in rats has found that SELK is regulated by dietary Se, while TXNRD2 is not (Barnes et al. 2009). Furthermore, although GPX1, GPX2, and GPX4 are all predicted targets of $\mathrm{NMD}$, their respective responses to Se deficiency are very different (Bermano et al. 1996; Wingler et al. 1999; Seyedali and Berry 2014). It has been suggested that other factors, such as differential regulation of selenoprotein expression by EIF4A3 (Budiman et al. 2009) or dependence of selenocysteine insertion on two different Sec-tRNA isoforms (Jameson and Diamond 2004), could explain the apparent resistance of GPX4 to NMD under low Se; however, neither of these two mechanisms can explain the observed increased abundance of GPX2 mRNA.

In this study, we used a systems biology approach to explore the mechanisms behind the hierarchical regulation of selenoprotein mRNAs. A colon adenocarcinoma cell line (Caco-2) was chosen as the experimental model system since these cells have previously been found to exhibit appropriate hierarchical regulation (Pagmantidis et al. 2005) and dietary Se levels have been implicated in colorectal cancer risk (Hughes et al. 2015). Expression of selenoprotein mRNAs in Caco-2 cells grown under different Se conditions (from Se deficiency to Se repletion) was determined experimentally and used to build mathematical models of selenoprotein translation that incorporated the different processes likely to be involved in determining the selenoprotein mRNA hierarchy. Fitting the data to the models allowed us to predict the combined effects of NMD inhibition and Se deficiency and these predictions were tested experimentally following knockdown of UPF1, a crucial NMD factor (Kurosaki and Maquat 2013). We show that a single model, which includes competition among selenocysteine insertion, premature termination, and NMD, and deadenylation-dependent mRNA turnover can explain the observed variations in mRNA levels for all selenoprotein mRNAs studied with the exception of SPS2, which is likely regulated by a different mechanism.

\section{RESULTS}

\section{Effect of Se status on selenoprotein mRNA levels in Caco-2 cells}

To investigate the effects of Se status on selenoprotein mRNA levels in Caco-2 cells, RNA was extracted from cells grown either in media deficient in Se (NoSe) or in media supplemented with different concentrations of sodium selenite, ranging from $5 \mathrm{nM}$ (considered as sub-optimal Se supply on the basis of GPX1 expression [Crosley et al. 2007]) to $40 \mathrm{nM}$ (considered as adequate Se supply [Crosley et al. 2007]). Sodium selenite was chosen as the supplement since this has been found previously to provide a better source of Se for selenoprotein synthesis in cells in culture than selenate or selenomethioinine (Hoefig et al. 2011). Se depletion had little or no effect on cell viability as judged by the absence of morphological differences, growth rate, and time to reach confluence (within 5\%-10\% regardless of Se status) under microscopic observations. Eleven selenoprotein mRNAs were selected for analysis based on the proposed importance of the corresponding protein in colonic function and the ability to detect their expression in Caco-2 cells under all Se conditions. Expression levels were determined using RTqPCR (Fig. 1). Compared with expression in Se-deficient conditions, a $30 \%$ increase in total selenoprotein mRNA was observed at $5 \mathrm{nM}$ sodium selenite $(P<0.01$, ANOVA), followed by a plateau (Fig. 1A). Most of this increase was due to an increase of SELH, GPX4, and SEPW1 mRNA, while SPS2 mRNA contribution was negative (Fig. 1B). When mRNA levels were normalized to mRNA abundance in the Se-deficient medium, Se status had a marked effect on mRNA levels for some selenoproteins but not others (Fig. 1C). A large Se-dependent increase (250\%-350\%) in RNA abundance was observed for GPX1, SEPW1, and SELH in cells supplemented with $5 \mathrm{nM}$ sodium selenite, before reaching a plateau for added concentrations above $10 \mathrm{nM}$. A moderate increase $(130 \%-150 \%)$ was observed for TXNRD2 and GPX4 mRNA levels, which reached a plateau at around 10-20 $\mathrm{nM}$ supplemented sodium selenite. A similar increase in RNA level was observed for SEPP1 and TXNRD1 mRNA but only for concentrations of sodium selenite of $20 \mathrm{nM}$ and above. On the contrary, increased Se supply had little or no effect on SELK and SEP15 mRNA levels. In addition, a 30\% decrease in RNA levels was observed for GPX2 and SPS2 mRNA when the medium was supplemented with 10 nM sodium selenite or more compared with Se-deficient medium. As a result, under these conditions, GPX1 and SEPW1 mRNAs appeared to be the lowest in the hierarchy, being the most sensitive to reduced Se supply, SELK and SEP15 were high in the hierarchy with no changes in expression associated with difference in Se supply, whereas both GPX2 and SPS2 
A

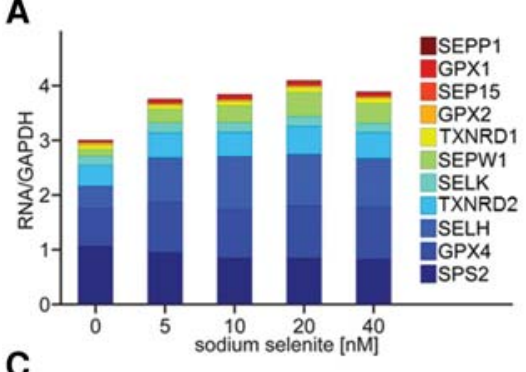

C

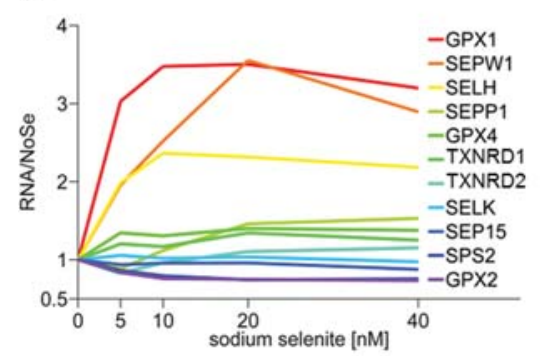

B
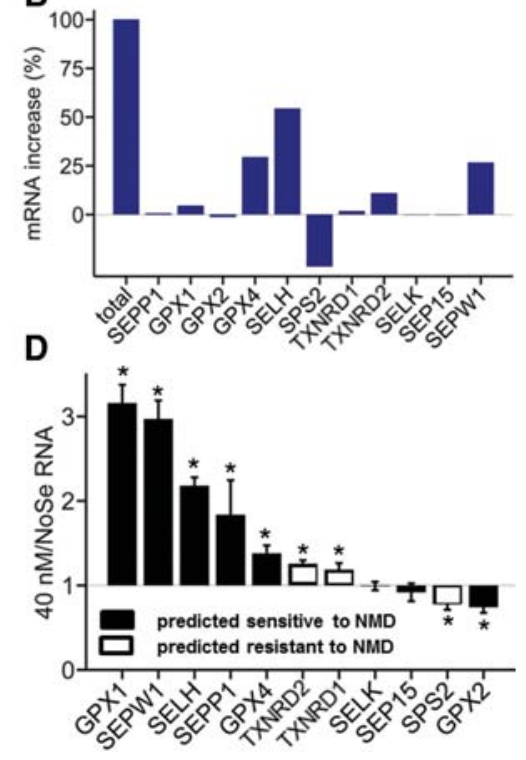

FIGURE 1. Selenoprotein mRNA abundance in Caco-2 cells grown in media supplemented with different sodium selenite concentrations. (A) Total selenoprotein mRNA abundance normalized to GAPDH. (B) Contribution of increase of individual selenoprotein mRNAs to the total increase in selenoprotein mRNA in high Se supplementation $(40 \mathrm{nM})$ over no added Se (NoSe). $(C)$ Selenoprotein mRNA abundance, normalized to mRNA abundance obtained for NoSe. $(D)$ Ratio of selenoprotein mRNA abundance between high Se supplementation (40 nM) Se and NoSe. All data were produced from eight individual experiments $(n=8)$ run in duplicates. $(*)$ $P<0.05$, $t$-test.

responded differently in showing increased mRNA expression when Se supply was limited.

Among selenoprotein mRNAs experimentally observed as sensitive to Se supply, most were predicted to be targets of NMD, according to the 50- to 55-nt NMD rule (Fig. 1D). However, SEP15 and GPX2, both predicted to be NMD targets, showed no response or a reduction in mRNA abundance, respectively, to increased Se supply. Similarly, the abundance of most selenoprotein mRNAs predicted to be resistant to NMD did not respond to changes in Se availability, except for TXNRD1 and TXNRD2 mRNA, which showed a rise in response to increased Se supply in the Caco-2 cell model. There was no correlation between selenoprotein mRNA abundance and sensitivity to Se $(\rho=0.17, P=0.61$; Materials and Methods), indicating that mRNA abundance itself does not influence the selenoprotein hierarchy.

\section{Mathematical models of selenoprotein translation can fit the experimental data}

To further explore the extent to which NMD alone could explain the effects of Se status on mRNA expression of different selenoproteins, a simple process-like mathematical model of selenoprotein translation (Model 1, Fig. 2A) was built and fitted to the experimental data. The model included the following reactions: transcription, translation initiation, translation elongation until the Sec-encoding UGA codon, binding of tRNASec to the transcript, either NMD or inser- tion of Sec and dissociation of tRNAsec, elongation from UGA to stop codon and termination, and background degradation of the mRNA (Fig. 2A). The same model structure and initial conditions were used for modeling translation of all selenoproteins; however, the process rates were chosen according to known selenoprotein transcript features (e.g., the rate of translation from UGA to stop codon depended on the length of individual mRNAs from the UGA to the stop codon) or were used for fitting the model (Supplemental Table S1; Ingolia et al. 2011; Schwanhäusser et al. 2011; Trcek et al. 2013).

Model 1 included the competition between detection of a PTC (followed by immediate NMD) and selenocysteine insertion (in essence, competition between binding of a release factor and binding of tRNAsec to the ribosome), and it provided a very good fit to the experimental mRNA abundance data for most selenoproteins; however, for GPX2 and SPS2, experimental data deviated substantially from the predicted model for all data points (Fig. 3; Supplemental Table S3). In Model 1, more available Se leads to more Sec insertion and less NMD-mediated degradation, and as a result, the model predicts that only an increase in mRNA abundance is possible under such conditions. Since the experimental data show that this is not the case, the results suggest that although competition between NMD-mediated degradation and Sec-insertion can account for some of the observed effects, in particular for selenoproteins lower in the hierarchy, it cannot account for the inverse dependence of mRNA abundance on Se abundance featured by GPX2 and SPS2.

Although a good fit of a model to experimental data supports its validity, there is the chance of underdetermination -when several different parameter values of a model lead to the same goodness of fit. Indeed this was the case for the nonresponsive SELK and SEP15, where Model 1 provided a good fit, but uncertainty of the inferred model parameters was high. Potentially, this uncertainty would weaken interpretation of additional modeling work on SELK and SEP15 and therefore we left these transcripts out of further analysis.

As Model 1 did not fit all the experimental data, further models of selenoprotein translation were developed in which additional mechanisms that affect selenoprotein hierarchy were taken into consideration. A potential weakness of Model 1 was that it modeled each selenoprotein independently. As translation of all selenoproteins is dependent on Sec-insertion, at low Se levels selenoproteins high in the hierarchy have been proposed to compete with 

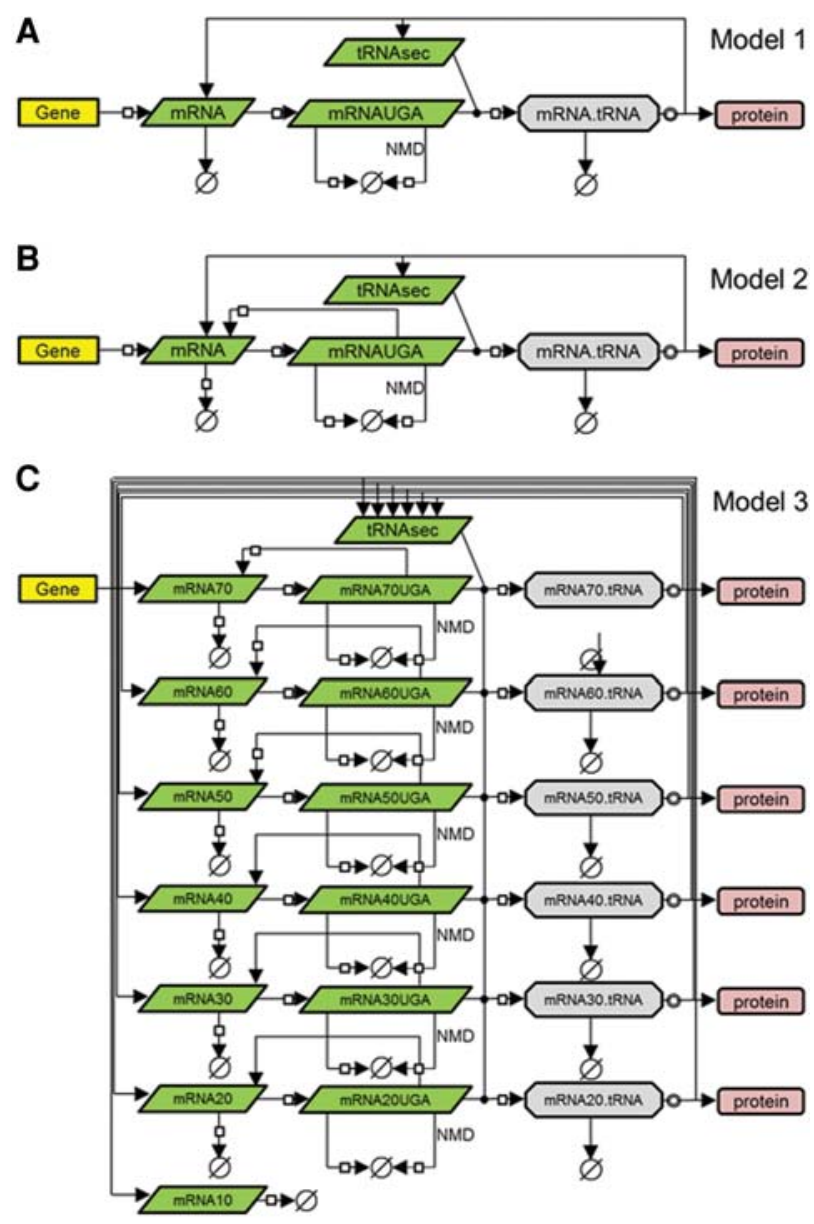

$\rightarrow$ association $\rightarrow$ dissociation $\rightarrow$ process $\varnothing \quad$ sink

FIGURE 2. Mathematical models of selenoprotein translation in SBGN (Le Novère et al. 2009). All models include the following processes: gene transcription, ribosome binding at the initiation codon, elongation until the Sec-encoding UGA codon, binding of Sec-tRNA to the transcript, NMD of mRNA, dissociating of Sec-tRNA, elongation up to stop codon and termination of translation, degradation of the mRNA. (A) Model 1: competition between NMD and selenocysteine insertion. (B) Model 2: competition among NMD, selenocysteine insertion, and ribosome drop-off. $(C)$ Model 3: competition among NMD, selenocysteine insertion, and ribosome drop-off, plus poly(A)-dependent mRNA degradation. In all models it is assumed that a constant pool of aminoacylated tRNAsec is available.

selenoproteins low in the hierarchy for the binding of tRNASec, causing them to undergo NMD. We modified Model 1 to incorporate aspects of competition (Model 1B, which is in essence an integration of eleven different versions of Model 1, one for each selenoprotein) (Low et al. 2000). Model 1B did not provide any improvement over Model 1 (data not shown), therefore additional models were tested.

Model 2 takes into account evidence suggesting that detection of a PTC does not always lead to NMD (Buchan and Stansfield 2007), but can also lead to normal ribosomal termination (ribosome drop-off). Therefore in this model, the competition between NMD and selenocysteine insertion is kept, but the outcome of PTC detection can either be NMD or ribosome drop-off, which leads to a free mRNA molecule (Model 2, Fig. 2B; Supplemental Table S3). However, addition of this new reaction did not improve the fit. Importantly, since Model 2 also did not include a mechanism that would stabilize the mRNA at low Se levels, we were still unable to match the experimental data for GPX2 and SPS2.

Model 3 accounts for the fact that mRNA degradation is strongly determined by the length of the poly(A) tail of the mRNA (Model 3, Fig. 2C; Cao and Parker 2001; Funakoshi et al. 2007). In Model 3, each transcript is associated with a poly(A) tail (model adapted from Cao and Parker 2001). Each successful selenoprotein translation shortens the poly(A) $(70 \rightarrow 60 \rightarrow \ldots \rightarrow 10$; a.u.), until at a critical size mRNA degradation is triggered. If the ribosome drops off at the premature UGA, poly(A) length is maintained. While Model 3 brought no improvement of fit for most selenoproteins, it was able to fit experimental data for SPS2 and GPX2 better than Model 1 (Model 1/GPX2: $\mathrm{BIC}=16.52$; Model 1/SPS2: 16.20; Model 3/GPX2: 15.68; Model 3/SPS2: 15.75) (Figs. 2, 4; Supplemental Table S3). Therefore, while for the other selenoproteins the competition between NMD and selenocysteine insertion is sufficient to explain the sensitivity to Se, SPS2 and GPX2 seem to require the additional mechanism of ribosome drop-off and poly(A) tail lengthdependent mRNA degradation which prevents the shortening of the poly(A) tail during translation and thus increases stability of the transcript. This suggests that for SPS2 and GPX2, ribosome drop-off occurs more frequently than NMD, while for the other selenoproteins NMD is the more frequent occurrence.

\section{Model validation with NMD knockdown}

Our results suggested that the selenoprotein hierarchy can be partly explained by competition among NMD, termination without NMD, and selenocysteine insertion. Therefore, changing any one of these three processes should affect each selenoprotein differently. To experimentally validate Model 3, expression of the helicase up-frameshift 1 (UPF1), a major component of the NMD process, was knocked down using siRNA technology. mRNAs that were either highly sensitive to changes in Se supply (GPX1 and $S E P W 1)$, moderately sensitive (GPX4), or display a decrease in abundance when Se supply increases (GPX2 and SPS2), were selected to evaluate the role of NMD on selenoprotein mRNA levels.

First, model predictions were generated for different levels of UPF1 knockdown (KD), under the assumption that the rate of NMD decreases linearly with increased level of UPF1 KD and that $100 \%$ UPF1 KD should lead to an absence of the NMD process. For the five selected selenoproteins, the model predicted an increase in mRNA abundance with UPF1 KD, with the largest increase for GPX2 and SPS2 

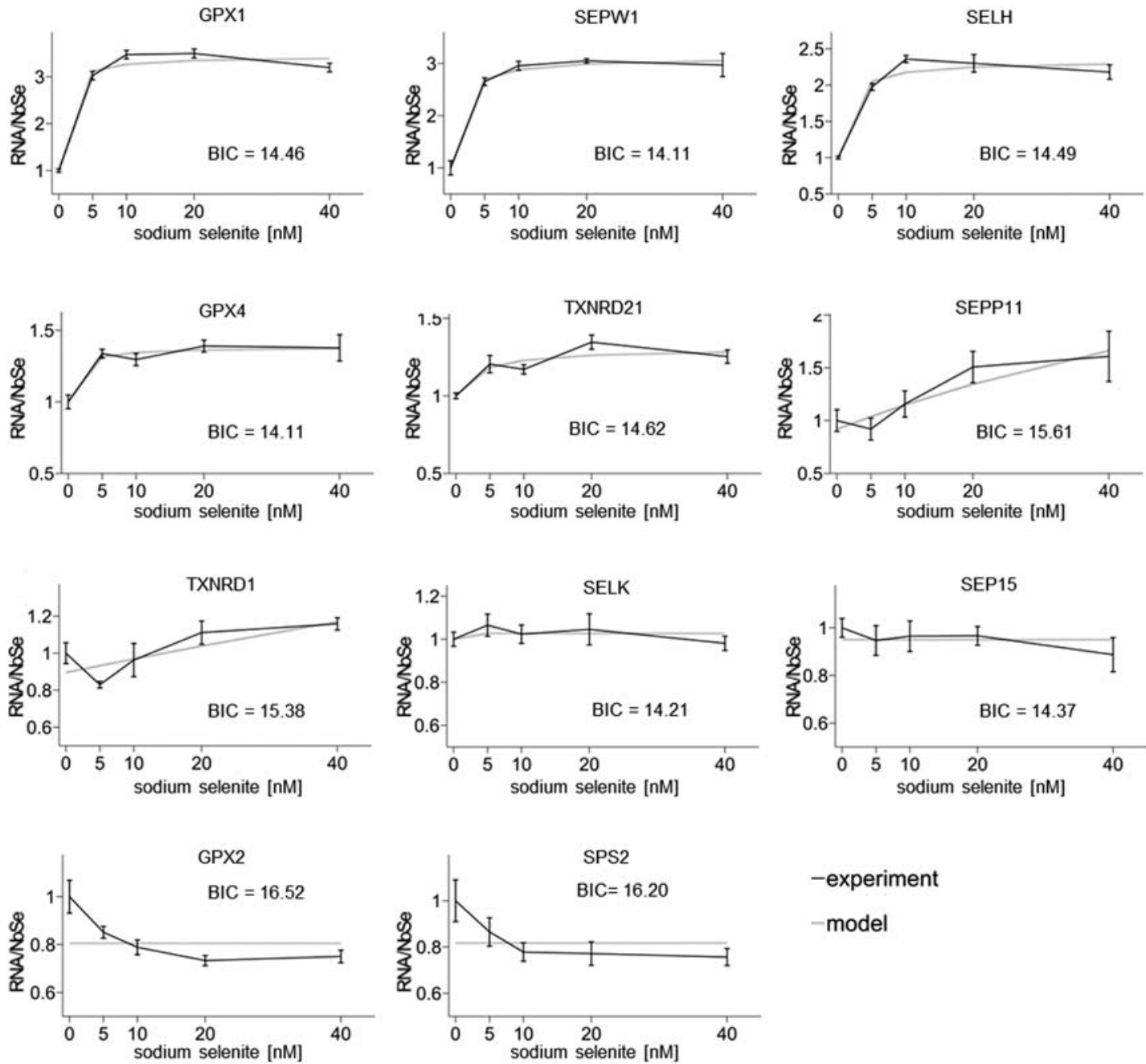

FIGURE 3. Best Model 1 fit to the experimental data for each individual selenoprotein. The quality of the model fit is given by the Bayesian information criterion (BIC) (see Materials and Methods).

without added sodium selenite at $100 \% \mathrm{KD}(\sim 80$-fold and $\sim 30$-fold, respectively). In comparison, the predicted increase for GPX1, GPX4, and SEPW1 at zero-added selenite and $100 \% \mathrm{KD}$ was only approximately threefold. The model also predicted that the differences between different levels of UPF1 KD for each selenoprotein would be highest for zero-added sodium selenite and would gradually disappear with higher added sodium selenite concentrations.

Model predictions were tested using RTqPCR to determine the effect of UPF1 KD on selenoprotein mRNA abundance for different Se content. siRNA silencing was performed separately in cells grown in the absence of Se or in culture media supplemented with either $5 \mathrm{nM}$ or $40 \mathrm{nM}$ sodium selenite. Efficiency of UPF1 mRNA KD was 25\% after $48 \mathrm{~h}$ treatment with siRNA and $52 \%$ after
$72 \mathrm{~h}$, as assessed by quantifying UPF1 mRNA expression compared to nonspecific KD control. The quantified selenoprotein mRNA abundances were compared with the modeling prediction at the same level of UPF1 KD (e.g., for a $\mathrm{KD}$ of $25 \%$, we imposed a reduction of $25 \%$ of the NMD rate in the model). The effects of UPF1 KD on GPX1, GPX2, GPX4, and SEPW1 are as predicted by the model (Fig. 5). In contrast, the
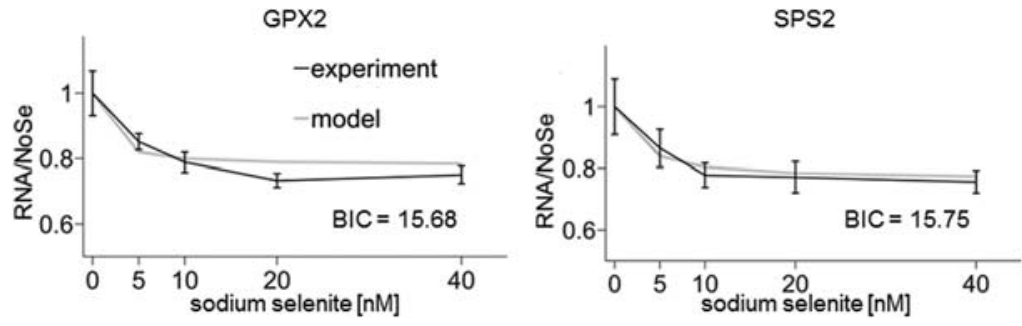

FIGURE 4. Best Model 3 fit to the experimental data for GPX2 and SPS2. The quality of the model fit is given by the Bayesian information criterion (BIC). 

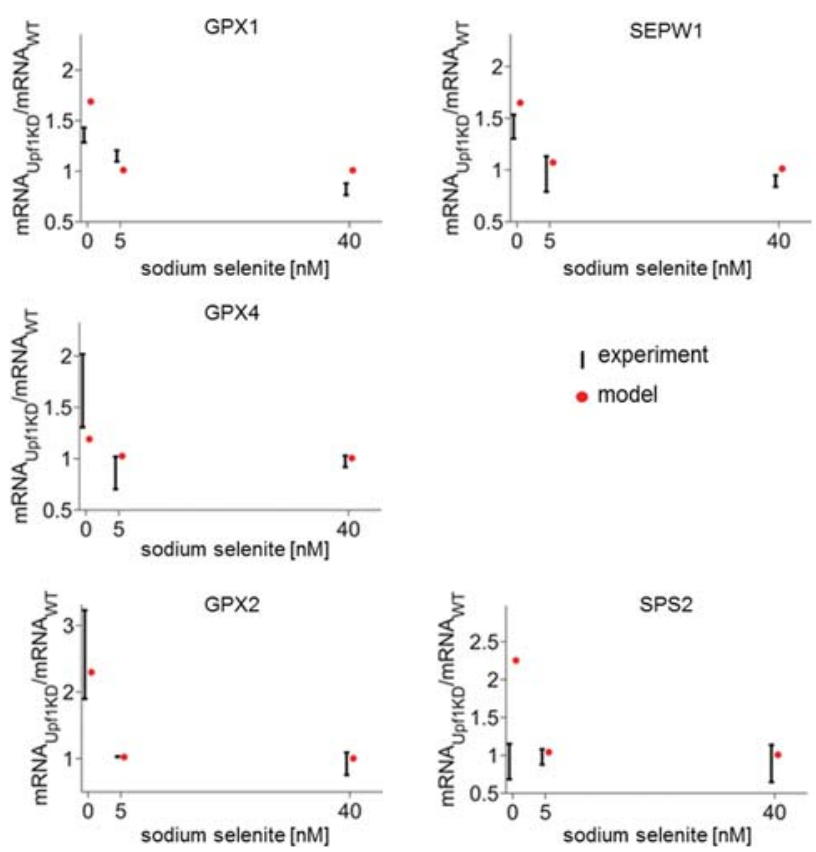

FIGURE 5. Selenoprotein mRNA abundance after UPF1 KD. Experimental results are given in black as mean \pm S.D. $(n=2$, S.D. uncorrected), while the model predictions are given by red dots.

model predictions did not match the experimentally observed SPS2 mRNA abundances, which were found not to be sensitive to UPF1 KD (Fig. 5). This indicates that Model 3 offers a plausible explanation for the observed selenoprotein mRNA hierarchy for most selenoproteins, but not for SPS2, which requires a different mechanism to explain the sensitivity of its mRNA expression to Se.

\section{DISCUSSION}

Although the phenomenon of the hierarchical regulation of selenoprotein mRNA expression under limited Se availability is well documented (Wingler et al. 1999; Hesketh 2008), the mechanisms behind it are not yet fully understood (Sunde and Raines 2011). The results of the present study are for most selenoprotein mRNAs compatible with a competition among three processes that can occur when the ribosome reaches the internal UGA codon: selenocysteine insertion, premature translation termination, and NMD. The exception was SPS2, which is regulated by another mechanism. While we do not provide an explanation for the special status of SPS2 among the selenoproteins, its role in the synthesis of selenocysteine and thus in regulation of its own protein synthesis and the synthesis of all the other selenoproteins might be the underlying cause.

Recent evidence, combined with earlier studies, strongly indicates that competition between NMD and selenocysteine insertion plays a crucial role in regulation of selenoprotein expression: (i) Either by in vitro transcription experiments (Toyoda et al. 1990; Bermano et al. 1995; Wingler et al.
1999) or by measuring both nuclear and cytoplasmic transcripts abundance (Moriarty et al. 1998), it has been shown that regulation of selenoprotein expression by Se is neither due to transcriptional regulations nor to mRNA processing, but occurs at the level of selenoprotein translation and/or mRNA stability; (ii) predicted susceptibility of transcripts to NMD, according to the currently accepted models of mammalian NMD, correlates with sensitivity to Se deficiency (Seyedali and Berry 2014); (iii) knocking down SMG1, a factor that was shown to be required for NMD, eliminates selenoprotein mRNA sensitivity to Se deficiency (Seyedali and Berry 2014); and (iv) increasing Se content increases the stability of selenoprotein mRNAs (Seyedali and Berry 2014). Our experimental results support and are consistent with those of Seyedali and Berry. Although they used a different cell model (HEK293T), the sensitivities of different selenoproteins to Se status in both studies are compatible, with the exception of GPX4, which was found to be insensitive to changes in Se supply and to NMD in HEK293T cells but to be moderately sensitive to both in Caco 2 cells. This could reflect a difference in the selenoprotein hierarchy between cells of kidney and colon origin. Previously, GPX4 has been found to be moderately sensitive to Se supply in several other cell types including liver (Bermano et al. 1995). An alternative explanation for the discrepancy is that the known GPX4 isoforms could be differentially susceptible to NMD, and that not the same isoform dominates in different cell types. In the present study, the primer for GPX4 recognized all GPX4 isoforms. For SPS2, both studies concluded that NMD cannot explain its sensitivity to Se status.

The present study provides both computational and experimental models that support a role of NMD in the control of hierarchical regulation of selenoprotein mRNAs and are consistent with the hypothesis that competition between NMD and selenocysteine insertion plays a crucial role in selenoprotein expression regulation. Another combined experimental and computational study before ours was unable to offer a model explanation (NMD competition with selenocysteine insertion) of the increase in GPX2 abundance under low Se conditions (Wingler et al. 1999). We show here that by including normal termination as the third competing process, GPX2 measurements can be explained by our computational model. By using the same model to predict the effects of NMD inhibition on the mRNA abundances of different selenoproteins and experimentally verifying the predictions, we provide additional validation of the model for all tested selenoproteins, except SPS2 (Fig. 5).

Indirect support for the presented model (competition between NMD, Sec insertion and normal termination) also derives from recent ribosome profiling studies. If there is substantial normal termination at the UGA codon, one would expect that the ribosome density (obtained by ribosome profiling, a measure of the presence of translating ribosome on transcripts) for selenoproteins would be higher before the UGA codon and lower after it. This was observed 
in two recent studies (Ingolia et al. 2011; Howard et al. 2013). While it is possible that the observed change in ribosome density could be the result of NMD, recent measurements show that NMD in the cytoplasm is an inherently quick process that probably would not be detected with ribosome profiling (Trcek et al. 2013). Additionally, in our recent study where we looked for changes in ribosome profiles across the genome, NMD targets were not identified (Zupanic et al. 2014).

It is important to stress that in our work we have only tested the suitability of the NMD hypotheses to explain the hierarchical regulation of selenoprotein mRNAs and showed that it explains all experimental measurements (except for SPS2), but we did not test any of the competing explanations of the hierarchy. Another proposed mechanism is the use of two different tRNAsec isoforms in selenocysteine insertion by selenoprotein mRNAs and a change in expression of one of the isoforms when Se supply is low (Howard et al. 2007, 2013). Although an effect of the tRNAsec isoforms has been demonstrated, it was rather small, and it is not clear how it could be used to explain the rather large change in mRNA abundance observed at low Se concentrations. Another possible mechanism is the recently discovered regulation of selenoprotein by miRNAs: mi-185 has been shown to have an effect on GPX2 and SPS2. Since NMD cannot explain the experimental observations for SPS2, it is possible that miRNA regulation is the dominant mechanism for this selenoprotein (Maciel-Dominguez et al. 2013).

Our study did not take into account the specific molecular mechanisms behind the hierarchy. In the future, the interactions between cis-regulatory features, such as the SECIS, sequence surrounding the UGA and SREs, and selenoprotein translation factors, such as SBP2, EEFSEC, NCL, SECP43, EIF4A3, and tRNAsec (Low et al. 2000; Small-Howard et al. 2006; Squires et al. 2007; Budiman et al. 2009; Fixsen and Howard 2010; Caban and Copeland 2012; GonzalezFlores et al. 2012), should be included in the model and independently tested. Further work is required to relate our model of how NMD is related not only to the hierarchical regulation of selenoprotein mRNAs but also to the higher level of expression of the encoded proteins.

Our model suggests that NMD is inefficient for GPX2, while the positions of the stop codon with respect to the intron suggests otherwise. Thus, the results suggest that selenoprotein mRNAs do not play by the canonical rules of what constitutes a good target for NMD. We propose two possible solutions: either selenoprotein mRNAs in general do not conform to the rules of NMD or that the competition with selenocysteine insertion (not present for other NMD targets) and normal termination (which could very well be affected by the selenocysteine insertion machinery) render it apparently less efficient.

In conclusion, our results show that a single mechanism, namely competition among the selenocysteine insertion, NMD, and normal termination, can explain the hierarchical regulation of selenoprotein mRNAs, with the exception of SPS2. Experiments that would measure the length of the poly(A) chain of the selenoproteins during with and without NMD KD would be able to provide definite proof (Cao and Parker 2003). It is interesting to speculate that the competition among selenocysteine insertion, NMD, and normal termination could also be responsible for the different hierarchies of selenoproteins in different tissues, e.g., GPX4 mRNA was shown to increase with Se in liver, but decrease in muscle. It has been shown that Se levels differ between tissues (Schomburg and Schweizer 2009) and so does NMD (Zetoune et al. 2008); however, a systematic comparison of both has not yet been performed in a single organism.

\section{MATERIALS AND METHODS}

\section{Cell culture and treatment}

Human colon adenocarcinoma cells (Caco-2) were grown at $37^{\circ} \mathrm{C}$ in a $5 \% \mathrm{CO}_{2}$ atmosphere in Dulbecco's modified essential medium with $4.5 \mathrm{~g} / \mathrm{L}$ glucose and Glutamax (Invitrogen) supplemented with $10 \%$ fetal calf serum (Sigma), $1 \%$ (v/v) penicillin-streptomycin (Invitrogen), and 1\% nonessential amino acid (Invitrogen). For depletion supplementation experiments, cells were transferred $1 \mathrm{~d}$ after being passaged to a serum-free DMEM medium containing $0.1 \%(\mathrm{v} / \mathrm{v})$ penicillin-streptomycin, $1 \%(\mathrm{v} / \mathrm{v})(100$ units $/ \mathrm{mL})$, insulin $(5 \mu \mathrm{g} / \mathrm{mL})$, and transferrin $(5 \mu \mathrm{g} / \mathrm{mL})$ without (Se-deficient medium) or supplemented with sodium selenite to provide an equivalent of 5-40 nM Se-repleted medium. After $3 \mathrm{~d}$ culture in Se-deficient or Se-supplemented medium, as used previously (Pagmantidis et al. 2005), cells were harvested and total RNA extracted. Microscopic observation was used to assess the effects of various Se levels on cell viability.

\section{siRNA-mediated down-regulation of UPF1}

Knockdown of expression of UPF1 was achieved by transient transfection of Caco-2 cells with a pool of four distinct siRNA (SI03120432, SI02629963, SI00045605, SI00045598) targeting UPF1 (QIAGEN) or with AllStars Negative Control siRNA (QIAGEN) in the presence of Hi-Perfect transfection reagent according to manufacturer's protocol. After 48-72 h, cells were harvested and RNA extracted.

\section{RNA extraction and reverse transcription}

Total RNA was extracted from Caco-2 cells as described in Pagmantidis et al. (2008). Briefly, culture medium was removed, cells were washed twice in ice-cold PBS, harvested and homogenized in $1 \mathrm{~mL}$ TRIzol reagent (Ambion, Paisley), and then frozen overnight at $-80^{\circ} \mathrm{C}$. After thawing on ice, TRIzol was removed by adding $0.2 \mathrm{~mL}$ chloroform $/ \mathrm{mL}$ of TRIzol and samples centrifuged for 15 min, $12000 \mathrm{~g}, 4^{\circ} \mathrm{C}$. To increase the purity of the RNA, this was followed by an extraction in $0.4 \mathrm{~mL}$ of phenol:chloroform:isoamyl alcohol $(25: 24: 1) / \mathrm{mL}$ of TRIzol and centrifugation for $15 \mathrm{~min}$, $12,000 \mathrm{~g}, 4^{\circ} \mathrm{C}$. Phenol was removed by addition of $0.2 \mathrm{~mL}$ chloroform, gentle vortexing and centrifugation for $15 \mathrm{~min}, 12,000 \mathrm{~g}$, 
$4^{\circ} \mathrm{C}$. Finally, RNA was precipitated by incubating the upper phase in $0.5 \mathrm{~mL}$ isopropanol per $\mathrm{mL}$ TRIzol for $30 \mathrm{~min}$ on ice followed by centrifugation for $30 \mathrm{~min}, 12,000 \mathrm{~g}, 4^{\circ} \mathrm{C}$. The pellet was washed in $75 \% \mathrm{DNase} / \mathrm{RNase}$-free $\mathrm{EtOH}$, dried for $5 \mathrm{~min}$ in vacuum desiccator and finally resuspended in $40 \mu \mathrm{L}$ of DNase/RNase free water and quantified by nanodrop and RNA quality assessed by the $A_{260} / A_{280}$ ratio.

Reverse transcription of $0.5 \mu \mathrm{g}$ total RNA was carried out using Transcriptor Reverse Transcriptase kit (Roche) in the presence of 100 pmol oligo(dT), according to the manufacturer's protocol. Reverse transcripts were frozen at $-20^{\circ} \mathrm{C}$ immediately after synthesis.

\section{Real-time quantitative PCR}

The qPCR reactions were performed in duplicate using $2 \mu \mathrm{L}$ of 50 fold (or fivefold for UPF1 siRNA knockdown) diluted cDNA in 20 $\mu \mathrm{L}$ reaction mixtures using SYBR Green Master mix (QIAGEN) used as a fluorescent reporter, and selenoprotein specific primers (250 nM for each primer; see Supplemental Table S2). The qPCR reactions included an initial denaturation $\left(95^{\circ} \mathrm{C}, 5 \mathrm{~min}\right)$, followed by 45 cycles of amplification (denaturation: $95^{\circ} \mathrm{C}, 10 \mathrm{sec}$; touchdown annealing from $55^{\circ} \mathrm{C}$ to $60^{\circ} \mathrm{C}$ depending on primer annealing temperature, $15 \mathrm{sec}$; elongation: $72^{\circ} \mathrm{C}, 5 \mathrm{sec}$ ), and a melt curve analysis (from $65^{\circ} \mathrm{C}$ to $95^{\circ} \mathrm{C}$ ) followed by a rapid cooling step at $40^{\circ} \mathrm{C}$ for $30 \mathrm{sec}$. The specificities of the PCR amplifications were assessed by examination of the melt curves to confirm the presence of single gene-specific peaks. Standard curves (five standards in duplicate) were generated for each amplicon from serial dilution of purified PCR products corresponding to the same sequence amplified using a RotorGene Q platform. Absolute quantification of RNA was achieved by comparing the fluorescence of samples with the amplification of the standards and normalized to GAPDH.

\section{Mathematical modeling and statistics}

Mathematical models of selenoprotein translation (see Results) were built and simulated in PottersWheel (Maiwald and Timmer 2008) and are available as Supplemental Material. The model reactions and their rates for fixed parameters and physiological ranges for the free parameters were chosen from the literature and can be found in Supplemental Table S1. In particular, transcription and RNA degradation rates were taken from the global gene expression data set in mouse from Schwanhäusser et al. (2011), translation rates were taken from a ribosome profiling study in mice of Ingolia et al. (2011), and NMD-related rates were taken from measurements of the NMD rates in Trcek et al. (2013). The free model parameters were fitted to the experimental measurements of selenoprotein mRNA abundance in PottersWheel, iterating between the trust region algorithm and the genetic algorithm. The fitting was run at least a hundred times for each model/experimental data combination. The quality of the fit was evaluated using the Bayesian information criterion (BIC). BIC is based on the likelihood function and includes penalization of the number of model parameters (if two models fit the data equally well, the model with fewer parameters is preferred).

We tested the robustness of the model fitting using identifiability analysis. Briefly, identifiability analysis tells whether there is a single set of model parameters that best described the data or many differ- ent parameter sets that produce the same quality of fit. In this case, the obtained parameter values need to be taken with caution and further validation is required. We ran the identifiability analysis for each model/experimental data combination in PottersWheel using the profile likelihood method, as described by Raue et al. (2009).

Correlation between ranked mRNA abundance and rank in the selenoprotein hierarchy was evaluated using the Spearman's rank correlation coefficient. The abundance ranking was based on mRNA abundance at $10 \mathrm{nM}$ supplemented sodium selenite, while the selenoprotein hierarchy ranking was based on the ratio of mRNA abundance between $40 \mathrm{nM}$ and $0 \mathrm{nM}$ sodium selenite supplementation.

\section{SUPPLEMENTAL MATERIAL}

Supplemental material is available for this article.

\section{ACKNOWLEDGMENTS}

A.Z., C.M., J.E.H., and D.P.S. thank the Biotechnology and Biological Sciences Research Council (BBSRC) for support (BB/ H011471/1). G.V.B.H. was supported by a scholarship from Coordination for the Improvement of Higher Education Personnel (CAPES).

Received December 23, 2015; accepted April 25, 2016.

\section{REFERENCES}

Barnes KM, Evenson JK, Raines AM, Sunde RA. 2009. Transcript analysis of the selenoproteome indicates that dietary selenium requirements of rats based on selenium-regulated selenoprotein mRNA levels are uniformly less than those based on glutathione peroxidase activity. J Nutr 139: 199-206.

Bermano G, Nicol F, Dyer JA, Sunde RA, Beckett GJ, Arthur JR, Hesketh JE. 1995. Tissue-specific regulation of selenoenzyme gene expression during selenium deficiency in rats. Biochem $J$ 311: 425-430.

Bermano G, Arthur JR, Hesketh JE. 1996. Role of the $3^{\prime}$ untranslated region in the regulation of cytosolic glutathione peroxidase and phospholipid-hydroperoxide glutathione peroxidase gene expression by selenium supply. Biochem J 320: 891-895.

Berry MJ, Harney JW, Ohama T, Hatfield DL. 1994. Selenocysteine insertion or termination: factors affecting UGA codon fate and complementary anticodon:codon mutations. Nucleic Acids Res 22: 3753-3759.

Buchan J, Stansfield I. 2007. Halting a cellular production line: responses to ribosomal pausing during translation. Biol Cell 99: 475-487.

Budiman M, Bubenik J, Miniard A, Middleton L, Gerber C, Cash A, Driscoll D. 2009. Eukaryotic initiation factor $4 \mathrm{a} 3$ is a selenium-regulated RNA-binding protein that selectively inhibits selenocysteine incorporation. Mol Cell 35: 479-489.

Caban K, Copeland P. 2012. Selenocysteine insertion sequence (SECIS)binding protein 2 alters conformational dynamics of residues involved in tRNA accommodation in $80 \mathrm{~S}$ ribosomes. $\mathrm{J}$ Biol Chem 287: 10664-10673.

Cao D, Parker R. 2001. Computational modeling of eukaryotic mRNA turnover. RNA 7: 1192-1212.

Cao D, Parker R. 2003. Computational modeling and experimental analysis of nonsense-mediated decay in yeast. Cell 113: 533-545.

Copeland PR, Fletcher JE, Carlson BA, Hatfield DL, Driscoll DM. 2000. A novel RNA binding protein, SBP2, is required for the translation of mammalian selenoprotein mRNAs. EMBO J 19: 306-314. 
Crosley LK, Meplan C, Nicol F, Rundlof AK, Arner ESJ, Hesketh JE, Arthur JR. 2007. Differential regulation of expression of cytosolic and mitochondrial thioredoxin reductase in rat liver and kidney. Arch Biochem Biophys 459: 178-188.

Fixsen SM, Howard MT. 2010. Processive selenocysteine incorporation during synthesis of eukaryotic selenoproteins. J Mol Biol 399: 385-396.

Fletcher JE, Copeland PR, Driscoll DM, Krol A. 2001. The selenocysteine incorporation machinery: interactions between the SECIS RNA and the SECIS-binding protein SBP2. RNA 7: 1442-1453.

Funakoshi Y, Doi Y, Hosoda N, Uchida N, Osawa M, Shimada I, Tsujimoto M, Suzuki T, Katada T, Hoshino S. 2007. Mechanism of mRNA deadenylation: evidence for a molecular interplay between translation termination factor eRF3 and mRNA deadenylases. Genes Dev 21: 3135-3148.

Gonzalez-Flores JN, Gupta N, DeMong LW, Copeland PR. 2012. The selenocysteine-specific elongation factor contains a novel and multi-functional domain. J Biol Chem 287: 38936-38945.

Hesketh J. 2008. Nutrigenomics and selenium: gene expression patterns, physiological targets, and genetics. Annu Rev Nutr 28: 157-177.

Hoefig CS, Renko K, Kohrle J, Birringer M, Schomburg L. 2011. Comparison of different selenocompounds with respect to nutritional value vs. toxicity using liver cells in culture. J Nutr Biochem 22: 945-955.

Howard MT, Moyle MW, Aggarwal G, Carlson BA, Anderson CB. 2007. A recoding element that stimulates decoding of UGA codons by Sec tRNA $^{[\mathrm{Ser}] \mathrm{Sec}}$. RNA 13: 912-920.

Howard MT, Carlson BA, Anderson CB, Hatfield DL. 2013. Translational redefinition of UGA codons is regulated by selenium availability. J Biol Chem 288: 19401-19413.

Hughes DJ, Fedirko V, Jenab M, Schomburg L, Meplan C, Freisling H, Bueno-de-Mesquita HB, Hybsier S, Becker NP, Czuban M, et al. 2015. Selenium status is associated with colorectal cancer risk in the European prospective investigation of cancer and nutrition cohort. Int J Cancer 136: 1149-1161.

Ingolia NT, Lareau LF, Weissman JS. 2011. Ribosome profiling of mouse embryonic stem cells reveals the complexity and dynamics of mammalian proteomes. Cell 147: 789-802.

Jameson RR, Diamond AM. 2004. A regulatory role for Sec tRNA ${ }^{[\mathrm{Ser}] \mathrm{Sec}}$ in selenoprotein synthesis. RNA 10: 1142-1152.

Kryukov GV, Castellano S, Novoselov SV, Lobanov AV, Zehtab O, Guigó R, Gladyshev VN. 2003. Characterization of mammalian selenoproteomes. Science 300: 1439-1443.

Kurosaki T, Maquat L. 2013. Rules that govern UPF1 binding to mRNA 3' UTRs. Proc Natl Acad Sci 110: 3357-3362.

Labunskyy VM, Hatfield DL, Gladyshev VN. 2014. Selenoproteins: molecular pathways and physiological roles. Physiol Rev 94: 739-777.

Latrèche L, Jean-Jean O, Driscoll D, Chavatte L. 2009. Novel structural determinants in human SECIS elements modulate the translational recoding of UGA as selenocysteine. Nucleic Acids Res 37: 5868-5880.

Le Novère N, Hucka M, Mi H, Moodie S, Schreiber F, Sorokin A, Demir E, Wegner K, Aladjem M, Wimalaratne SM, et al. 2009. The systems biology graphical notation. Nat Biotechnol 27: 735-741.

Low SC, Grundner-Culemann E, Harney JW, Berry MJ. 2000. SECISSBP2 interactions dictate selenocysteine incorporation efficiency and selenoprotein hierarchy. EMBO J 19: 6882-6890.

Maciel-Dominguez A, Swan D, Ford D, Hesketh J. 2013. Selenium alters miRNA profile in an intestinal cell line: evidence that miR-185 regulates expression of GPX2 and SEPSH2. Mol Nutr Food Res 57: 2195-2205.

Maiwald T, Timmer J. 2008. Dynamical modeling and multi-experiment fitting with PottersWheel. Bioinformatics 24: 2037-2043.

Moriarty PM, Reddy CC, Maquat LE. 1998. Selenium deficiency reduces the abundance of mRNA for Se-dependent glutathione peroxidase 1 by a UGA-dependent mechanism likely to be nonsense codon-mediated decay of cytoplasmic mRNA. Mol Cell Biol 18: 2932-2939.

Pagmantidis V, Bermano G, Villette S, Broom I, Arthur J, Hesketh J. 2005. Effects of Se-depletion on glutathione peroxidase and selenoprotein W gene expression in the colon. FEBS Lett 579: 792-796.

Pagmantidis V, Méplan C, van Schothorst EM, Keijer J, Hesketh JE. 2008. Supplementation of healthy volunteers with nutritionally relevant amounts of selenium increases the expression of lymphocyte protein biosynthesis genes. Am J Clin Nutr 87: 181-189.

Popp M, Maquat L. 2013. Organizing principles of mammalian nonsense-mediated mRNA decay. Annu Rev Genet 47: 139-165.

Raue A, Kreutz C, Maiwald T, Bachmann J, Schilling M, Klingmüller U, Timmer J. 2009. Structural and practical identifiability analysis of partially observed dynamical models by exploiting the profile likelihood. Bioinformatics 25: 1923-1929.

Rayman MP. 2012. Selenium and human health. Lancet 379: 12561268.

Schomburg L, Schweizer U. 2009. Hierarchical regulation of selenoprotein expression and sex-specific effects of selenium. Biochim Biophys Acta 1790: 1453-1462.

Schwanhäusser B, Busse D, Li N, Dittmar G, Schuchhardt J, Wolf J, Chen W, Selbach M. 2011. Global quantification of mammalian gene expression control. Nature 473: 337-342.

Seyedali A, Berry MJ. 2014. Nonsense-mediated decay factors are involved in the regulation of selenoprotein mRNA levels during selenium deficiency. RNA 20: 1248-1256.

Small-Howard A, Morozova N, Stoytcheva Z, Forry EP, Mansell JB, Harney JW, Carlson BA, Xu X-M, Hatfield DL, Berry MJ. 2006. Supramolecular complexes mediate selenocysteine incorporation in vivo. Mol Cell Biol 26: 2337-2346.

Squires JE, Stoytchev I, Forry EP, Berry MJ. 2007. SBP2 binding affinity is a major determinant in differential selenoprotein mRNA translation and sensitivity to nonsense-mediated decay. Mol Cell Biol 27: 7848-7855.

Sun XL, Li XJ, Moriarty PM, Henics T, LaDuca JP, Maquat LE. 2001. Nonsense-mediated decay of mRNA for the selenoprotein phospholipid hydroperoxide glutathione peroxidase is detectable in cultured cells but masked or inhibited in rat tissues. Mol Biol Cell 12: 1009-1017.

Sunde RA, Raines AM. 2011. Selenium regulation of the selenoprotein and nonselenoprotein transcriptomes in rodents. Adv Nutr 2: $138-150$.

Toyoda H, Himeno S, Imura N. 1990. Regulation of glutathione peroxidase mRNA level by dietary selenium manipulation. Biochim Biophys Acta 1049: 213-215.

Trcek T, Sato H, Singer R, Maquat L. 2013. Temporal and spatial characterization of nonsense-mediated mRNA decay. Genes Dev 27: 541-551.

Weiss S, Sunde R. 1997. Selenium regulation of classical glutathione peroxidase expression requires the $3^{\prime}$ untranslated region in Chinese hamster ovary cells. J Nutr 127: 1304-1310.

Wingler K, Böcher M, Flohé L, Kollmus H, Brigelius-Flohé R. 1999. mRNA stability and selenocysteine insertion sequence efficiency rank gastrointestinal glutathione peroxidase high in the hierarchy of selenoproteins. Eur J Biochem 259: 149-157.

Zetoune AB, Fontaniere S, Magnin D, Anczukow O, Buisson M, Zhang CX, Mazoyer S. 2008. Comparison of nonsense-mediated mRNA decay efficiency in various murine tissues. BMC Genet 9: 83.

Zupanic A, Meplan C, Grellscheid SN, Mathers JC, Kirkwood TBL, Hesketh JE, Shanley DP. 2014. Detecting translational regulation by change point analysis of ribosome profiling data sets. RNA 20: 1507-1518. 

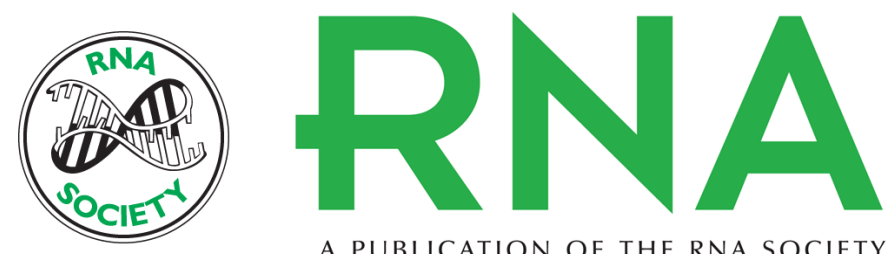

A PUBLICATION OF THE RNA SOCIETY

\section{Modeling and gene knockdown to assess the contribution of nonsense-mediated decay, premature termination, and selenocysteine insertion to the selenoprotein hierarchy}

Anze Zupanic, Catherine Meplan, Grazielle V.B. Huguenin, et al.

RNA 2016 22: 1076-1084 originally published online May 20, 2016

Access the most recent version at doi:10.1261/rna.055749.115

Supplemental Material

References

Open Access

Creative Commons License

Email Alerting Service
http://rnajournal.cshlp.org/content/suppl/2016/05/20/rna.055749.115.DC1

This article cites 49 articles, 25 of which can be accessed free at: http://rnajournal.cshlp.org/content/22/7/1076.full.html\#ref-list-1

Freely available online through the RNA Open Access option.

This article, published in RNA, is available under a Creative Commons License (Attribution 4.0 International), as described at http://creativecommons.org/licenses/by/4.0/.

Receive free email alerts when new articles cite this article - sign up in the box at the top right corner of the article or click here.
||||||| Providing Precise Solutions for your research.

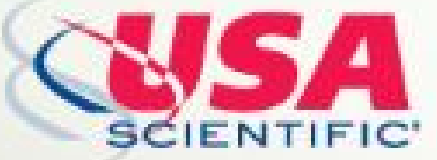

To subscribe to $R N A$ go to:

http://rnajournal.cshlp.org/subscriptions

(C) 2016 Zupanic et al.; Published by Cold Spring Harbor Laboratory Press for the RNA Society 